\title{
Transient CRISPR immunity leads to coexistence with phages
}

2

3 Sean Meaden ${ }^{1 *}$, Loris Capria ${ }^{1}$, Ellinor Alseth ${ }^{1}$, Ambarish Biswas $^{2}$, Luca Lenzi ${ }^{3}$, Angus

4 Buckling ${ }^{1}$, Stineke van Houte ${ }^{1}$, Edze R Westra ${ }^{1 *}$

5

6 1. Biosciences, University of Exeter, TR10 9EZ, UK

7 2. Department of Microbiology and Immunology, University of Otago, Dunedin, New

8 Zealand

9 3. Institute of Integrative Biology, University of Liverpool, L69 7ZB, UK

$10 *$ corresponding authors

11 email: s.meaden@exeter.ac.uk; e.r.westra@exeter.ac.uk 


\section{Abstract}

Phages play a major role in shaping the composition, evolution and function of bacterial communities. While bacteria and phages coexist in many natural environments, their coexistence is often short-lived in the lab due to the evolution of phage resistance. However, fitness costs associated with resistance and mutational loss of resistance alleles may limit the durability of acquired resistances, potentially allowing phages to re-invade the population. Here, we explore this idea in the context of bacteria that evolve CRISPR-based immunity against their phages. Consistent with previous studies, we found that the bacterium Pseudomonas aeruginosa PA14 evolved high levels of CRISPR-based immunity and low levels of surface-based resistance following infection with phage DMS3vir, which led to rapid phage extinction. However, when these pre-immunized bacterial populations were subsequently challenged with the same phage, they failed to clear the infection and instead stably coexisted with the phage. Analysis of bacterial genotypes and phenotypes over time explained why CRISPR-Cas immunity provides only a transient advantage: in the absence of phage (i.e. following the initial phage extinction) formerly CRISPR-immune bacteria regain sensitivity due to evolutionary loss of spacers, whereas in the presence of phage (i.e. upon reinfection) selection favours surface-based resistance over CRISPR immunity. The latter results from an infection-induced fitness cost of CRISPR-immunity that is due to phage gene expression prior to target DNA cleavage by the immune system. Together, these results show that CRISPR-Cas immune systems provide only a transient benefit to bacteria upon phage infection and help to explain why bacteria and phages can coexist in natural environments even when bacteria carry CRISPR-Cas adaptive immune systems that allow for rapid acquisition of immunity against phages. 
40 Phages are ubiquitous in prokaryotic communities and play a vital role in key ecological

41 processes. For example, phage predation may cause up to $20 \%$ of mortality of all bacteria in the world's oceans, making them key players in global nutrient cycling (Suttle 2007). In the deep terrestrial biosphere, phages regulate microbial communities- in turn shaping resource turnover (Daly et al. 2019) and in wetland ecosystems, phages have been associated with regulation of methanogens and sulfate reducers (Dalcin Martins et al. 2018). In addition to ecological processes, co-evolution between phages and bacteria can generate and accelerate increases in bacterial and phage genetic diversity (van Houte et al.; Paterson et al. 2010; Scanlan et al. 2015), mediate horizontal gene transfer (Canchaya et al. 2003) and alter competition among bacteria (reviewed in Koskella \& Brockhurst 2014). As well as this important ecological role, phages are also increasingly seen as a promising alternative to antibiotics to treat bacterial infections of plants, animals and humans (Wagenaar et al. 2005; Balogh et al. 2010; Kutter et al. 2010). Therefore, understanding the factors that shape the ecology and evolution of bacteria-phage interactions is fundamentally important.

The impact of phages on bacterial species and communities in ecological and biomedical settings is inevitably constrained by both pre-existing (innate) and acquired phage resistance (reviewed in Labrie et al. 2010). Bacteria can acquire phage resistance either by mutation or masking of the cell surface receptors to which the phages bind, or through their adaptive immune systems known as CRISPR-Cas (Clustered Regularly Interspaced Short Palindromic Repeats; CRISPR-associated). CRISPR-Cas works by inserting phage-derived sequences into

61 CRISPR loci on the host genome, which function as genetic memory to detect and destroy 62 phages carrying the same sequence (reviewed in Marraffini 2015). This can lead to rapid 
phage extinction, as phages are effectively being actively removed from the environment (as opposed to surface-based resistance where phages persist in the environment).

65

While CRISPR immunity can drive phages extinct in the short term, it is less clear how this immune system impacts phage persistence in the long term. Given that acquired resistance alleles are heritable, one would perhaps expect that pre-immunized bacterial populations would remain fully resistant to reinfection by the same phage genotype. On the other hand, there may be selection against CRISPR immunity due to costs in the form of autoimmunity (Stern et al. 2010), or if CRISPR is less effective under high phage exposure (Westra et al. 2015). Additionally recent theory and data suggest that individual bacteria may lose their CRISPR-resistance due to mutations in the cas genes or CRISPR arrays (Weissman et al. 2019).

To test the durability of CRISPR-based immunity, we recurrently infected Pseudomonas aeruginosa PA14 with phage DMS3vir (Cady et al. 2012), a mu-like phage (Budzik et al. 2004). By sequentially re-introducing the phage at different time intervals we vary the intensity of selection for resistance (surface mutation) and immunity (CRISPR) and track the resulting ecological dynamics. By using a combination of experimental evolution and

81 genomic analyses we tease apart the population genetics that underpin this host-parasite 82 interaction. These results have implications for how genetic diversity can be both generated and lost in bacterial populations and help explain the coexistence of bacteria and phages in natural microbial communities. 


\section{Results}

Populations lose the ability to drive phages extinct

CRISPR mediated immunity acts as an acquired, heritable genetic memory against phages (Marraffini 2015), however the timescales over which it is effective are unknown. Based on our mechanistic understanding of CRISPR immunity, one would predict that population-level immunity is long-lived. However, the few empirical studies that directly measure population and evolutionary dynamics of CRISPR-phage interactions have either focussed on short-term dynamics (Westra et al. 2015; van Houte et al. 2016) or examined CRISPR in isolation (Paez-Espino et al. 2013; Common et al. 2019), rather than the interplay between alternative resistance strategies. Recent theory and data suggests that resistance alleles may be lost from bacterial populations due to back mutations towards a sensitive phenotype when resistance is costly, which can in turn allow for phage persistence (Weissman et al. 2018; Chaudhry et al. 2018; Gurney et al. 2019). To test this, we exposed P. aeruginosa PA14, which carries a type I-F CRISPR system, to phage DMS3vir, which carries a mutation in its repressor gene that prevents lysogeny, as well as a partial protospacer match which promotes primed spacer acquisition by $P$. aeruginosa PA14 (Westra et al. 2015). We then measured whether populations could repeatedly drive phage DMS3vir to extinction following an initial phage exposure. Consistent with earlier findings, following the first infection treatment, phage titers were found to initially increase due to the phage epidemic that takes place in the sensitive

107 bacterial population, followed by a rapid decline in phage titers and extinction at 4 days post 108 infection (dpi) (Fig. 1A), due to the evolution of CRISPR-based immunity in the bacterial population (van Houte et al. 2016 and Fig. 2). To test whether these now immunized bacteria

110 could survive subsequent infections they were reinfected with $10^{7}$ pfu phage at 5 dpi.

111 Consistent with the bacterial population having evolved CRISPR-mediated phage resistance

112 in response to the earlier phage challenge the phage was rapidly driven to extinction (Fig. 
113 1B). Strikingly, when the same bacterial populations were re-infected 5 days later (at 10 dpi)

114 they had lost the ability to drive the phage extinct (Fig. 1B), and phage was able to persist for

115 the remainder of the 30-day experiment in all replicates of the same treatment. Similarly, in

116 the treatment where the first re-infection took place at $10 \mathrm{dpi}$, the bacterial populations also

117 had lost the ability to drive phage extinct (Fig. 1C). As expected, the treatment where phage

118 was added every day showed high phage titres throughout the experiment (Fig. 1D), and in

119 the phage-free treatment no phage could be detected (data not shown).

120

121

122

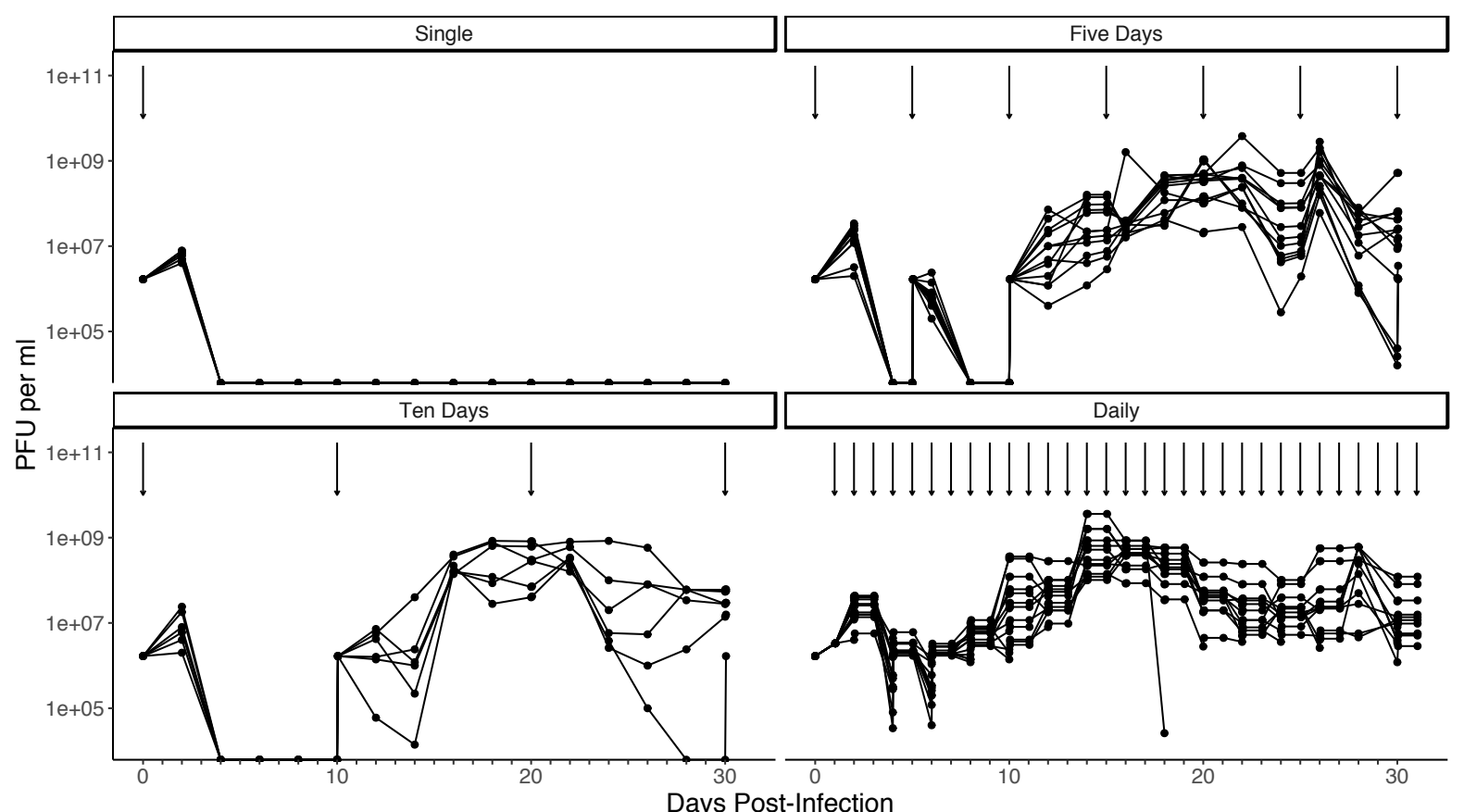

123

Figure 1. Phage titres over time upon infection of $P$. aeruginosa with DMS3vir. Arrows

126 denote timing of reinfection, each line represents an individual replicate population. A) No reinfection $(n=6)$. B) Reinfection every 5 days $(n=12)$. C) Reinfection every 10 days $(n=$ 6). D) Daily reinfection. $(\mathrm{n}=12)$. Phage titres were recorded every 2 days. Note this figure includes imputed values for non-recorded days that combine the previous days titre with the

130 phages that were added. Limit of detection is 200 PFU per $\mathrm{ml}$. 
Populations are invaded by surface resistance mutants and sensitive clones

133 To understand why phages can successfully re-infect populations that previously drove the

134 same phage extinct, we phenotypically characterised the composition of the host populations,

135 i.e. the proportion of sensitive bacteria as well as the proportion of bacteria that had acquired

136 at least one new spacer that conferred CRISPR-based immunity (CRISPR-immune herein) or

137 surface mutation based resistance (i.e. bacteria that evolved phage resistance through loss or

138 masking of the phage receptor, SM herein). At 4 dpi $0.66 \pm 0.036$ (mean proportion \pm SE) of

139 bacteria in the populations from the single infected treatment evolved CRISPR-immunity,

$140 \quad 0.25 \pm 0.040$ evolved SM resistance, and $0.083 \pm 0.022$ remained sensitive. At 30 dpi, these

141 fractions had changed to $0.0069 \pm 0.0069$ CRISPR immunity, $0.38 \pm 0.073 \mathrm{SM}$, and $0.61 \pm$

1420.73 sensitives. The invasion of sensitive bacteria in the absence of phage suggests that either

143 CRISPR immunity is associated with a constitutive fitness cost, or that there is back mutation

144 from CRISPR immunity to the sensitive, ancestral genotype (examined in detail later).

145 Next, to understand how the reinfection regimes impact the evolutionary dynamics of

146 the host population, we examined how the evolution of CRISPR immunity and surface

147 resistance varied across our treatments. At 4 dpi, we found an interaction between the

148 proportion of each phenotype and reinfection frequency (GLM, F1, $102=7.06, \mathrm{p}<0.0001)$,

149 with populations that were re-infected daily had slightly increased levels of SM compared to

150 those only infected once $\left(\mathrm{F}_{1,35}=17.94, \mathrm{p}<0.001\right)$, which is consistent with previous results

151 that demonstrate a shift towards SM when phage exposure is high (Westra et al. 2015).

152 Interestingly, for all treatments, we observed a significant reduction in CRISPR-immune

153 clones over time (quasibinomial GLM, $\mathrm{F}_{1,22}=179.8, \mathrm{p}<0.0001$ ), but no interaction between

154 treatment and time (quasibinomial GLM, $\mathrm{F}_{1,16}=2.4, \mathrm{p}=0.16$ ), suggesting the reinfection

155 regime was not driving the observed decline. However, while in the single infection treatment 
the decline in CRISPR-immune clones was due to the invasion of sensitive bacteria, we

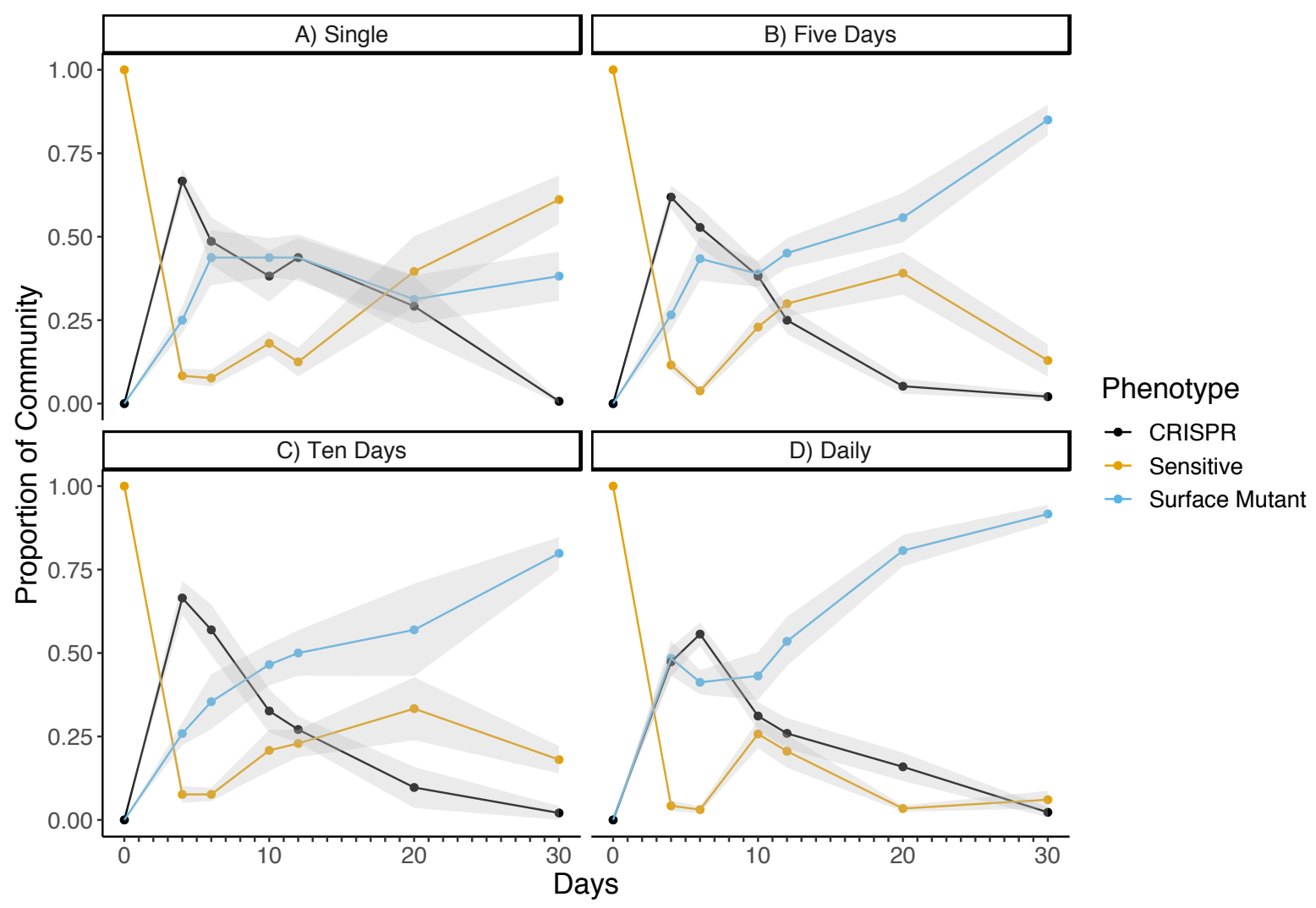

159 Figure 2. Frequencies of phage resistant and sensitive phenotypes. A) No reinfection, $\mathrm{n}=6$

160 B) Reinfection every 5 days, $\mathrm{n}=12 \mathrm{C}$ ) Reinfection every 10 days, $\mathrm{n}=6,5 \mathrm{D})$ Daily

161 reinfection, $\mathrm{n}=12$. Proportions were determined from phenotypic assays of 24 clones per population. Lines represent the means across populations and shaded areas represent \pm 1 SEM. CRISPR-immune bacteria are shown in black, bacteria with surface-based resistance in blue, and clones that remain sensitive to the phage in yellow.

The population genetics of phage resistance and immunity

167 These phenotypic data were further supported by genotypic data. First, we sequenced the genomes of a single SM clone from each population at day 12. Consistent with phage

169 DMS3vir being pilus-specific (Budzik et al. 2004), we identified in all cases a SNP in a pilus 170 affiliated gene (PilC, PilB, PilQ, PilY1), a deletion of the PilM gene or, most frequently, a 
$17110 \mathrm{~kb}$ deletion of 7 pilus-associated genes (at position 5948050 - 5958338; Fig. 3, panel E),

172 which was identical in all independent isolates and hence suggests these areas of the genome

173 contain sequences with microhomology that are prone to recombination. In addition, we

174 carried out deep sequencing of CRISPR array amplicons (CRISPR1 and CRISPR2) of all

175 experimental populations. This demonstrated an extremely high spacer diversity (133249

176 unique CRISPR arrays) that is sufficient to cover all 5643 possible target sequences on the

177 phage genome (Fig.S1). We found a significant interaction between time and reinfection

178 frequency, with spacer diversity declining in all groups with the exception of populations that

179 were infected daily, which maintained spacer diversity for longer (GLM, F $\mathrm{F}_{1,85}=6.65, \mathrm{p}<$

$180 \quad 0.001$, with qualitatively similar results for Shannon's or Simpson's diversity indices).

181 Moreover, the frequency of array sequences lacking any new spacers increased over time

182 (GLM, $\left.\mathrm{F}_{1,89}=228, \mathrm{p}<0.0001\right)$, as would be expected given the invasion of sensitive and SM

183 clones. Interestingly, arrays with multiple spacers all declined throughout the experiment (up

184 to 5 spacers, Fig 3, Panel A). When we tracked the fates of individual spacers (in order to

185 identify variation in spacer effectiveness) we typically found a consistent decline in spacer

186 abundance, with only a few exceptions (Fig 3, panel B) that most likely represent CRISPR

187 immune clones that subsequently acquired a surface mutation (Fig. 3, Panel C).

188 

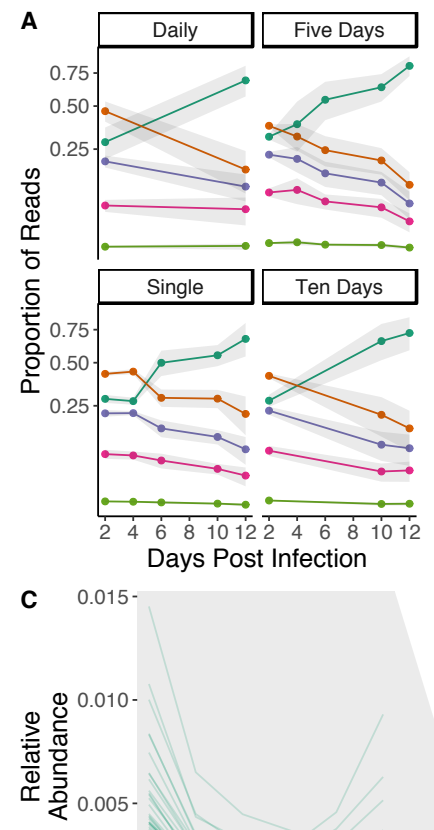

190

191 Figure 3. Population genetics of CRISPR arrays and surface mutants. A) Mean proportion of

192 reads with acquired spacers per treatment. Ribbon denotes 1 S.D. B) Decline in genetic

193 diversity in spacer arrays across treatments. These results are qualitatively similar regardless

194 of diversity index used (i.e. Shannon, Simpson's). C) Frequencies of individual arrays coverage of reads across the PA14 genome, for a single sample (S2), split by $1 \mathrm{~Kb}$ intervals.

Zoomed panel shows the 10,288 bp deletion containing pilus genes.
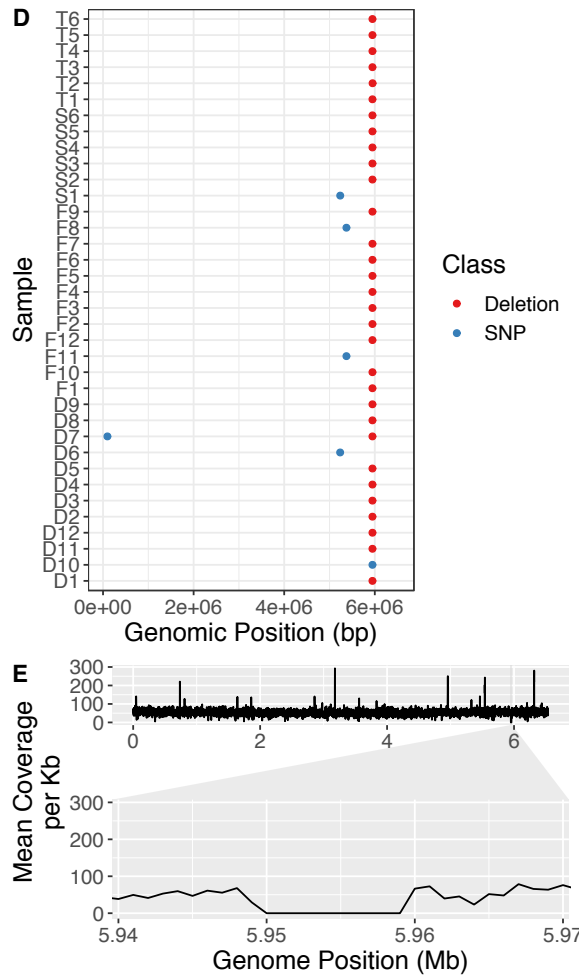

through time for a single population of the five-day reinfection regime. Orange line represents the ancestral array, with no new spacer. Green lines represent unique genotypes.

Zoomed panel shows individual spacer frequencies. Figures show the results for CR2, the more active locus, however these results are also representative of CR1. D) Whole genome analysis of re-sequenced clones exhibiting a surface modification phenotype at day 12- points denote SNP locations. Sample names denote infection regime and replicate. E) Mean Zoomed panel shows the 10,288 bp deletion containing pilus genes. 
205 The data discussed above show that CRISPR immunity dominates early on, but over time sensitive bacteria and SM clones invade. Since sensitive bacteria will act as a source for

207 phage, we hypothesised that their invasion may be responsible for the observed bacteria-

208 phage coexistence following re-infections at $10 \mathrm{dpi}$. Consistent with this hypothesis, bacterial

209 populations from 2 dpi were able to rapidly reduce phage DMS3vir titres whereas the same

210 populations from 12 dpi caused phage amplification $\left(\mathrm{GLM}, \mathrm{F}_{1,46}=172.29, \mathrm{p}<0.0001\right.$, Fig.

211 S2). Moreover, we found a strong interaction effect between phage titres and the proportion

212 of each phenotype in our experimental populations $\left(\mathrm{GLM}, \mathrm{F}_{1,627}=50.93, \mathrm{p}<0.0001\right)$.

213 Specifically, we found a positive correlation between phage titre and the frequency of

214 sensitive clones in the population $\left(\mathrm{GLM}, \mathrm{F}_{1,209}=12.3\right.$, adjusted $\mathrm{p}$-value $\left.<0.001\right)$, as well as a

215 negative correlation between phage titre and the frequency of CRISPR-immune clones

216 (GLM, $F_{1,209}, F=41.6$, adjusted p-value < 0.001) (Fig 4.). As expected, the proportion of

217 clones with SM resistance correlates less strongly with phage titre (as this phenotype neither

218 amplifies nor degrades phages). Taken together, these results are consistent with the idea that

219 CRISPR clones act as a 'sink' for phages whilst sensitive bacteria act as a 'source' for phages

220 and that the protective effect is lost as CRISPR-immune bacteria decline in frequency. 


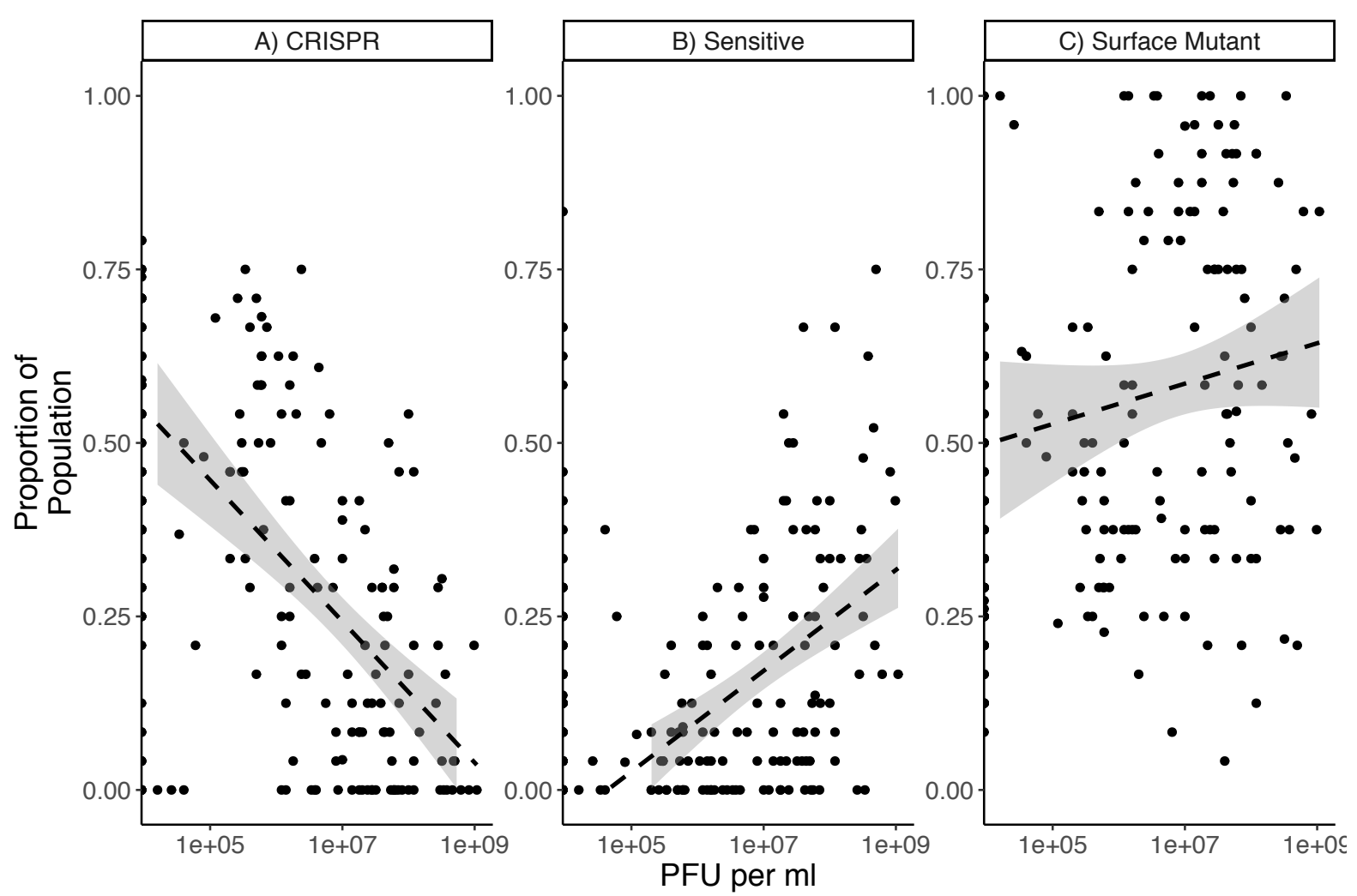

224 Figure 4. Correlations of each phenotype with phage titres throughout the experiment.

225 Dashed lines represent a linear model fit and shaded areas represent 95\% confidence intervals. A) Surface mutant bacteria, B) CRISPR-immune bacteria, C) Sensitive bacteria.

Phage densities drive the host evolutionary dynamics

229 Whereas invasion of sensitive bacteria can explain the phage population dynamics, it is less

230 clear what drives the invasion of sensitives in the first place. To understand this, we first

231 compared the host dynamics across the different infection regimes. Even though the decline

232 of CRISPR-immune clones was broadly similar across the various infection regimes, there

233 were clear differences in the invasion of sensitive bacteria and clones with SM resistance that

234 depended on the phage titers: sensitive bacteria were most likely to increase in frequency

235 when phages were absent, whereas SM tended to invade in the presence of phage (GLM,

236 interaction between phage presence and phenotype on change in frequency, $F_{1,611}=30.87, p$ 
$237<0.001$, Fig S3). Invasion of sensitives in the absence of phage may be driven by selection,

238 if sensitive bacteria are fitter than clones with CRISPR immunity under those conditions.

239 However, the observed increase in the frequency of sensitives may also result from back-

240 mutation from a CRISPR immune genotype to sensitive (for example mutations in cas genes

241 can inactivate the immune system (Jiang et al. 2013) or recombination between CRISPR

242 repeats can result in spacer loss (Lopez-Sanchez et al. 2012). To tease these processes apart,

243 we competed clones with different phenotypes isolated from these evolution experiments

244 against an ancestral reference strain in the absence of phages. We found that although the

245 clones did increase in fitness by day 12 (GLMM, main effect of time, $\chi^{2}=31.07, \mathrm{df}=6, \mathrm{p}<$

246 0.001, Fig. 5), there was no interaction between phenotype and time (GLMM, $\chi^{2}=3.11$, df $=$

$24711, \mathrm{p}=0.21)$ and no effect of treatment $\left(\mathrm{GLMM}, \chi^{2}=1.83, \mathrm{df}=9, \mathrm{p}=0.61\right)$. We also found

248 a borderline significant effect of phenotype (GLMM, $\left.\chi^{2}=6.54, \mathrm{df}=6, \mathrm{p}=0.038\right)$, however

249 post-hoc testing showed that there were no significant differences among phenotypes within

250 each timepoint (Table S1). When considered alongside the observed ecological dynamics, the

251 most parsimonious explanation is that sensitive bacteria invade the populations through the

252 loss of CRISPR immunity i.e. back-mutation where spacers are lost. This is most clear in the

253 single infection treatment where phages are absent: no phenotype has greater fitness yet

254 sensitive bacteria still invade (Fig. 2). 


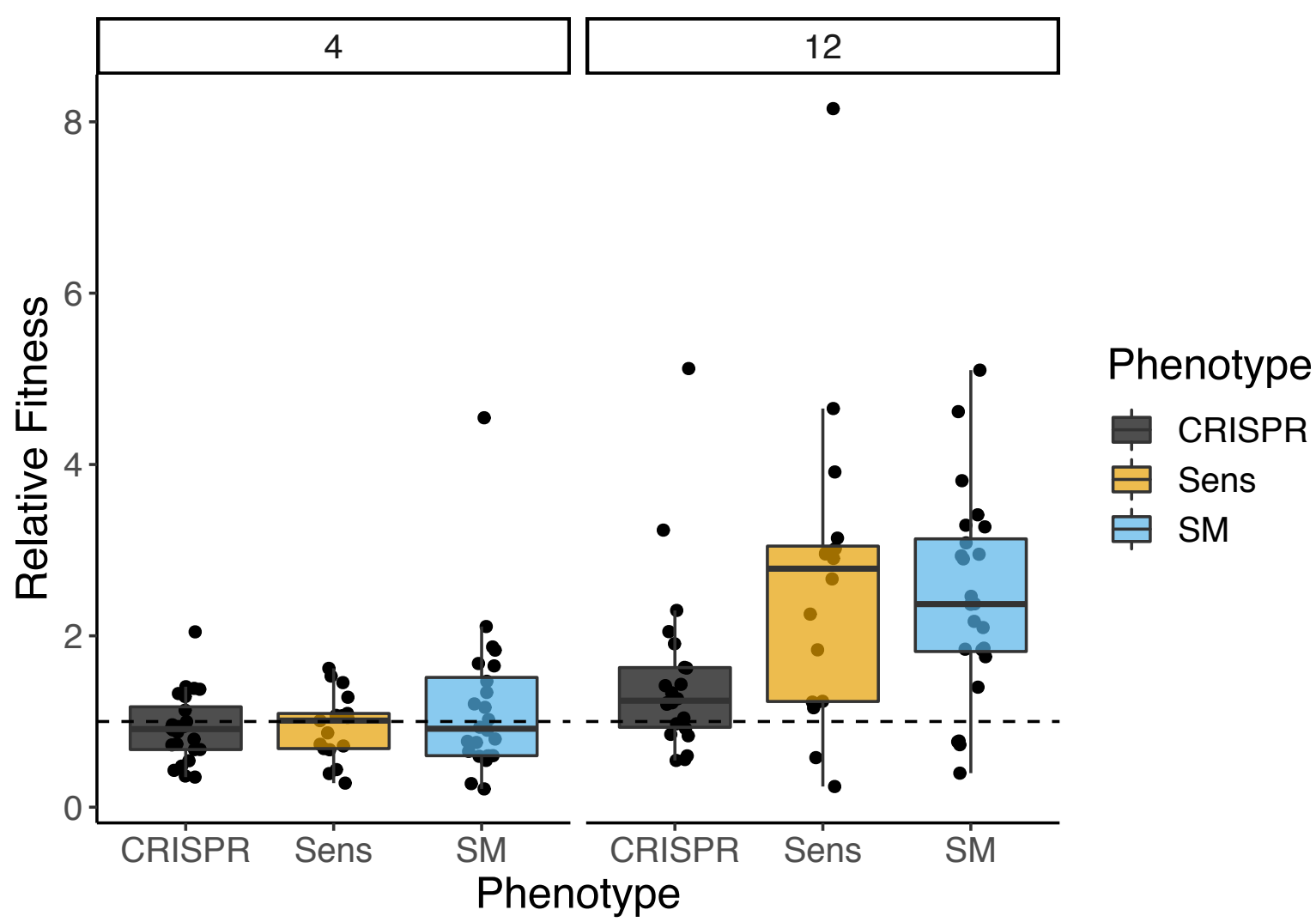

Fig. 5. Relative fitness of bacterial clones at early (day 4) and late (day 12) timepoints during the experiment (in the absence of phages). Relative fitness was determined from 3-day competition experiments against a marked reference strain in the absence of phages. Where possible, a clone of each phenotype was isolated from all populations. A) Clones isolated

261 from 4 DPI, B) Clones isolated from 12 DPI.

In contrast, the rapid invasion of SM clones observed in the evolution experiment, in the presence of phage, is likely explained by the fact that CRISPR immunity is associated with an inducible cost (i.e. a cost that increase during phage infections (Westra et al. 2015).

266 Indeed, a similar invasion of SM has been previously observed when a reservoir of sensitive

267 bacteria was introduced because the force of infection is increased (Chabas et al. 2016).

268 However, it has remained unclear what the mechanistic basis of this cost is. We envisaged 
three possible reasons for this inducible cost: (i) a cost of autoimmunity, where bacteria encoded genes in the period between infection (i.e. phage genome injection) and CRISPRmediated clearance of the phage. To explore the first hypothesis, we first performed a competition experiment between bacteria with CRISPR immunity that are unable to acquire novel spacers (i.e. a cas 1 deletion mutant) and a surface mutant. This competition was performed across a gradient of titres of DMS3mvir (a mutant of DMS3vir that is targeted by spacer1 in CRISPR array 2 of the PA14 $\Delta$ cas 1 strain; hence both the surface mutant and competing bacterial genotypes being insensitive to this phage). If the induced fitness cost of CRISPR-Cas immunity was due to the acquisition of self-targeting spacers, we would expect the cas 1 deletion mutant to outcompete the WT strain. However, this was not observed, with the cas 1 deletion mutant showing lower relative fitness than the WT strain $\left(\mathrm{GLM}, \mathrm{F}_{1,70}=\right.$ $17.85, \mathrm{p}<0.001$, Fig. S4). This suggests that the observed phage-induced fitness cost is not due to autoimmunity. Similarly, deep sequencing of bacterial populations that evolved phage resistance against DMS3vir showed that self-targeting with either zero $(\mathrm{n}=17)$ or a single mismatch $(n=18)$ to the host-genome spacers were very rare $(0.16 \%$ of all unique spacers $)$. However, one spacer was found to target both the phage and host genome with $100 \%$ sequence identity (hypothetical protein YP_950448.1 and DUF1804 family protein respectively). While this spacer was present in two populations, it was only observed at the first timepoint sampled (day 2), consistent with the idea that there is strong selection against a self-targeting spacer. Our results therefore suggest that although self-targeting is likely to be deleterious, it is insufficient to explain the initial invasion of SM clones. 
at 35, 60 and 120 minutes post-infection of a CRISPR immune clone that carries 2 spacers targeting DMS3vir (BIM2). BIM2 shows complete immunity to DMS3vir and no phage particles are produced following infection (data not shown; also see Landsberger et al. 2018). Differential expression analysis found no evidence that CRISPR-Cas expression is enhanced following infection (Fig. S5). Of all CRISPR-Cas genes, only Cas1 showed a significant difference, with slightly lower expression in infected BIM2 populations relative to controls $($ FDR adjusted $p$-value $=0.017, \log 2$ fold change $=-0.375)$. Finally, to test whether we could detect phage gene expression in the infected BIM2 populations we mapped reads to the DMS3vir genome. This revealed significant levels of genome-wide phage gene expression in the BIM2 populations, despite their CRISPR-based immunity. In total, phage expression in the BIM2 populations was around 5-fold lower compared to infected WT populations (Fig. (acr) and associated repressor ( $a c a$ ) expression (Fig. S6), which has previously been reported to be amongst the most strongly expressed genes on the phage DMS3vir genome (Stanley et al. 2019), and to a lesser degree, many other genes (Fig S7). This acr protein is specific for type I-E CRISPR-Cas systems and does not impact the type I-F CRISPR-Cas system encoded by PA14. Collectively, these data show that the phage is capable of expressing its genes prior to CRISPR mediated cleavage.

313 phage-induced fitness cost in CRISPR-immune bacteria, we next competed the BIM2 strain 314 and the surface mutant in the presence of either WT DMS3vir, an acr deletion mutant, and a mutant carrying a deletion of the entire acr operon. This revealed a similar fitness cost

316 regardless of the phage genotype (GLM, effect of genotype on relative fitness of BIM vs SM,

$317 \mathrm{~F}_{1,50}=2.15, \mathrm{p}=0.12$ ). However, given that many other phage genes are also expressed, albeit 318 at lower levels, the induced cost may well be due to expression of one or more other phage 
genes. We hypothesized that expression of the protease I gene might be particularly costly, since proteolytic activity could conceivably cause cytotoxicity in the cell. This essential gene is located immediately downstream of the $a c r$ and $a c a$ genes, and was found to be expressed both in this experiment as well as an independent RNAseq experiment using nanopore sequencing (data not shown). Given that a protease I deletion mutant would not be viable, we cloned and expressed the protease gene in WT P. aeruginosa to measure the cost of expression of this gene for the host. This showed that protease I expression reduced cell growth rates by approximately $13 \%$ relative to an empty vector control $\left(\mathrm{GLM}, \mathrm{F}_{1,46}=28.72\right.$, $p<0.001)$. Given that this is just one of the many genes expressed by the phage during infection it seems likely that phage gene expression prior to clearance of the infection is a source of the observed phage-induced cost of CRISPR-based immunity. However, we note that there may be additional unknown factors that contribute to an inducible-cost.

\section{Discussion}

335 Here we demonstrate that bacteria are rapidly immunised following phage infection and the

336 population is driving the phage to extinction. However, the success of this immunisation is

337 transient and successive re-introduction of the phage leads to stable coexistence between

338 bacteria and phages. This is due to the invasion of bacteria with SM resistance as well as

339 sensitive bacteria, which is caused by both an inducible fitness cost of CRISPR-based

340 immunity and the loss of spacers. We attribute the inducible cost to the phage gene

341 expression we observe which likely occurs prior to clearance of the infection by the CRISPR

342 immune system. Immune loss has been shown to result in the stable coexistence of bacteria 
and phages (Weissman et al 2018.; Chaudhry et al. 2018) and the loss of spacers, resulting in

344 a loss of immunity, is highly consistent the ecological dynamics we observe.

346 In a broader context, parasites are often invoked as a selective force for maintaining host

347 diversity through negative frequency dependent selection (Koskella \& Lively 2009).

348 However, the effectiveness of CRISPR-Cas in driving phage extinct shortly after the onset of

349 the phage epidemic (van Houte et al. 2016), removes this key mechanism for maintaining

350 host spacer diversity. Indeed, we observed that spacer diversity decreased in all treatments

351 (Fig. 3). This rapid decline in CRISPR diversity also helps to explain the observed

352 conservation of the trailer-end of CRISPR loci (Tyson \& Banfield 2007), although other

353 ecological and evolutionary processes, such as selective sweeps of multi-phage resistant

354 CRISPR clones (Weinberger et al. 2012), will also be important in natural communities.

355 Interestingly, we find that surface modifications that confer phage resistance in this

356 environment are not associated with a measurable trade-off in competitive fitness in the

357 absence of phage. Although the variation in the genetic basis of the SM phenotype (SNPs and

358 large deletions) suggests these mutations may vary in their fitness consequences in more

359 complex environments. For example, increasing the environmental complexity, in the form of

360 additional competitor species has been shown to amplify the associated fitness trade-off

361 (Alseth et al. 2019). Therefore, understanding these dynamics across a range of environments

362 will be of importance for future work.

364 The loss of spacer diversity over time is particularly relevant in the context of phage therapy,

365 which is experiencing renewed clinical interest (Dedrick et al. 2019). Long-lasting resistance

366 mechanisms may severely hamper therapeutic usage of phages, however this work shows that

367 one can take advantage of the loss of population-level CRISPR immunity over time and 
design well-timed re-infection schemes. Clinical trials where burn wound patients are treated

372 Kingwell 2015; Roach et al. 2017). This work also highlights an important potential issue associated with long-term information storage in CRISPR loci of bacterial populations

374 (Shipman et al. 2017; Shipman et al. 2016), since unique spacer sequences will inevitably be lost over time.

\section{Materials and Methods}

\section{Bacterial strains and phages}

The previously described $P$. aeruginosa strains UCBPP-PA14 and the isogenic mutant csy3::LacZ and phage DMS3vir have been previously described (Cady et al. 2012) and were used throughout this study.

\section{Evolution experiments}

384 Evolution experiments were performed in 6 replicates by inoculating $6 \mathrm{ml} \mathrm{M} 9$ supplemented with $0.2 \%$ glucose or LB with approximately $10^{6}$ bacteria from fresh overnight cultures of the WT strain and adding $10^{7} \mathrm{pfu}$ of DMS3vir, followed by incubation at $37^{\circ} \mathrm{C}$ while shaking at $180 \mathrm{rpm}$. Cultures were transferred daily 1:100 for 30 days. Reinfections with ancestral phage

$388\left(10^{7} \mathrm{pfu}\right)$ were performed as follows: a) bacterial cultures in the single infection treatment were only infected at $t=0 \mathrm{~b}$ ) bacterial cultures in the 5 day re-infection treatment were infected at $\mathrm{t}=0,5,10,15,20$ and $25 \mathrm{dpi}$ ) bacterial cultures in the 10 day re-infection

391 treatment were infected at $\mathrm{t}=0,10$ and 20 dpi d) bacterial cultures in the daily infection

392 treatment were infected at each daily transfer. 


\section{Phage extraction and titration}

395 Every second transfer, samples were taken just before transferring of the cultures into fresh

396 medium. Phage was isolated from these samples using chloroform extractions. Next, phage

397 was titrated by spotting serial dilutions of phage in M9 salts on a lawn of $P$. aeruginosa strain

UCBPP-PA14 csy3::LacZ bacteria for quantification.

399

400 Deep sequencing analysis

401 Full bacterial genomic DNA was isolated using the Qiagen QIAmp DNA mini kit as per the

402 manufacturer's protocols. A PCR amplification was performed for both CRISPR arrays

403 (CRISPR1 and CRISPR2, see table S2). PCR reactions contained $5 \mu$ l DreamTaq master mix

404 (ThermoScientific, UK), $0.5 \mu \mathrm{l}$ forward primer, $0.5 \mu 1$ reverse primer, $1.5 \mu 1$ MiliQ water, 0.5

$405 \mu \mathrm{LMSO}, 2 \mu \mathrm{l}$ template DNA. Sample purity was determined by NanoDrop and DNA

406 concentrations were quantified done using a Qubit fluorometer (ThermoFisher, UK).

407

408 CRISPR array sequencing protocol

409 Two separate CRISPR primer (CRISPR1 and CRISPR2 locus) pairs were designed for two

410 first round PCRs. $2 \mu$ of DNA entered a first-round of PCR. The primer design incorporates

411 a recognition sequence to allow a secondary nested PCR process. Samples were first purified

412 with Ampure SPRI Beads before entering the second PCR performed to incorporate Illumina

413 adapter sequences. Samples were purified using Ampure SPRI Beads before being quantified

414 using Qubit and assessed using the Fragment Analyzer. Successfully generated amplicon

415 libraries were taken forward and pooled in equimolar amounts, then size selected on a Pippin

416 prep using a range of $180-600 \mathrm{bps}$. The quantity and quality of each pool was assessed by

417 Bioanalyzer and subsequently by qPCR using the Illumina Library Quantification Kit from 
418 Kapa on a Roche Light Cycler LC480II according to manufacturer's instructions. Template

419 DNA was denatured according to the protocol described in the Illumina cBot User guide and

420 loaded at $12.5 \mathrm{pM}$ concentration. To help balance the complexity of the amplicon library

$421 \quad 15 \%$ PhiX was spiked in. The sequencing of each pool was carried out on one lane of an

422 Illumina MiSeq, at 2x250 bp paired-end sequencing with v2 chemistry.

423

424 Bioinformatics analysis

425 Sequence Quality Control

426 Base-calling and de-multiplexing of indexed reads was performed by CASAVA version 1.8.2

427 (Illumina) to produce 97 samples from each of the 2 lanes of sequence data. FASTQ files

428 were trimmed to remove Illumina adapter sequences using Cutadapt version 1.2.1 (Martin

429 2011). The option "-O 3" was set, so the 3" end of any reads which matched the adapter

430 sequence over at least $3 \mathrm{bp}$ was trimmed off. The reads were further trimmed to remove low

431 quality bases, using Sickle version 1.200 with a minimum window quality score of 20 . After

432 trimming, reads shorter than $10 \mathrm{bp}$ were removed. The raw reads were subjected to a

433 Cutadapt trimming step to remove PCR primer sequences that could potentially introduce an

434 artificial level of complexity in the samples. To improve base quality in both read pairs,

435 sequencing errors were corrected in both forward and reverse reads using the error-correct

436 module within SPAdes sequence assembler, version 3.1.0 (Bankevich et al. 2012). Read pairs

437 were aligned to produce a single sequence for each pair that would entirely span the amplicon

438 using PEAR (version 0.9.10; (Zhang et al. 2014)). Additionally, sequences with uncalled

439 bases (Ns) were removed. To remove sequences originating from potential PCR primer

440 dimers or from any spurious amplification events, a size selection was applied to each

441 merged sequence set, respectively between 30-140bp for CRISPR1 and 70-500bp for

442 CRISPR2. Fragmented PhiX phage genome was added to the sequence library in order to 
increase the sequence complexity. To remove any 'bleed through' of PhiX sequences, each

444 sample was compared with the complete PhiX sequence (GenBank gi9626372) using of the dataset.

\section{Clustering and diversity metrics}

449 For each dataset, any sequences passing the filters (from any sample) were merged into a

450 single file. This final sequence file, plus its own metadata file describing each sample, was

451 used for the analysis by using a custom pipeline based on QIIME 1.9.0 (Caporaso et al.

452 2010). Clusters were defined using SWARM (Mahé et al. 2014), using the strictest (default)

453 parameters. This tool aggregates a sequence to a cluster if the sequence shows similarity with

454 any of the sequences already present in that cluster. Importantly, the similarity threshold is not fixed but defined within the dataset. A minimum cluster size filter is applied to retain clusters containing at least 2 sequences and potential chimeric-sequences due to PCR events were discarded as well. To calculate the abundance of each cluster, sequences were then aligned on the centroid sequence identified for each clusters, using a minimum similarity threshold of $99 \%$ for the entire length of the sequence using the 'usearch_global' function in VSEARCH.

462 The sequencing depth of all samples was explored using the 'Chao 1' (Chao, 1984) richness 463 index plotted as a rarefaction curve. Counts in the cluster abundance tables were repeatedly 464 sub-sampled (rarefied; 33 repetitions) at sampling depths of 1000, 12000, 22000, .. 150000.

465 The average Chao1 value obtained by repeating the test 33 times is assigned as alpha-

466 diversity at that specific number of reads for that sample implemented in Qiime. Because all

467 samples reached a clear asymptote, i.e. no samples were under-sampled with regards to 
spacer diversity, rarefaction was not applied. An abundance table for each locus was used to estimate the richness and evenness of the samples using the following estimators: total observed sequence variants, Shannon, Simpson, Simpson evenness again conducted with Qiime.

Whole-genome sequencing

Populations from T12 were plated and 12 resulting colonies were screened for their phage laboratory stock was included. Each colony was then suspended in $100 \mathrm{ul} \mathrm{H}_{2} 0$ and streaked across an LB agar plate. Cells were scraped from these plates and added to bead beating tubes prior to shipping to the MicrobesNG service (Birmingham UK). Three beads were washed purified using an equal volume of SPRI beads and resuspended in EB buffer. DNA was quantified in triplicates with the Quantit dsDNA HS assay in an Ependorff AF2200 plate reader. Genomic DNA libraries were prepared using Nextera XT Library Prep Kit (Illumina, increased to $1 \mathrm{~min}$ from 30 seconds. DNA quantification and library preparation were carried out on a Hamilton Microlab STAR automated liquid handling system. Pooled libraries were quantified using the Kapa Biosystems Library Quantification Kit for Illumina on a Roche light cycler 96 qPCR machine. Libraries were sequenced on the Illumina HiSeq using a sliding window quality cutoff of Q15 (Bolger et al. 2014). The ancestral control was initially mapped to the PA14 reference genome (NC_008463) using the Breseq pipeline (version 
0.32.0, (Deatherage \& Barrick 2014). The reference genome was then modified to match the identified mutations (to account for divergence between the laboratory strain and original reference genome). The remaining samples were then mapped against this modified reference and alignments were used for identifying mutations.

\section{Immunity and resistance profiling}

501 Bacterial immunity against the ancestral phage was determined as described before (Westra et al. 2015; van Houte et al. 2016; Chabas et al. 2018) by streaking individual clones (24

503 clones per sample) through ancestral phage DMS3vir and phage DMS3vir carrying the anti-

504 CRISPR F1 (acrIF1) gene (Bondy-Denomy et al. 2012). Bacterial clones sensitive to both 505 phages were scored as "sensitive", those resistant to DMS3vir but sensitive to DMS3vir+AcrF1 were scored as "CRISPR immune", and bacterial clones resistant to both

507 phages were scored as "surface mutants". CRISPR-Cas-mediated immunity was further

508 confirmed by PCR using primers CTAAGCCTTGTACGAAGTCTC and

509 CGCCGAAGGCCAGCGCGCCGGTG (for CRISPR 1) and

\section{GCCGTCCAGAAGTCACCACCCG and TCAGCAAGTTACGAGACCTCG (for CRISPR}

511 2). Surface modification was further confirmed on the basis of colony morphology (since

512 phage DMS3vir is pilus-specific, surface mutants have motility defects, resulting in a

513 modified colony morphology) and a lack of new CRISPR spacers. From these analyses

514 fractions of each phenotype (sensitive, CRISPR immune, surface mutant) were calculated for 515 each replicate experiment. 
518 In most cases general linear models were used using the appropriate error structure and

519 model residuals were assessed for model fit. Significance was determined following stepwise

520 deletion of terms or through Tukey post-hoc testing with adjustment for multiple

521 comparisons. General linear mixed-effect models were used for the competition assays, with

522 population specified as a random effect, due to the non-independence of clones isolated from

523 the same populations. Mixed effect modelling was carried out using the 'Ime4' package with

524 post-hoc testing conducted with the 'emmeans' package. For gene expression analysis, data

525 were analysed with the DESeq2 pipeline using a parametric fit for the dispersion (Love et al.

526 2014). Statistical analyses were carried out in Rstudio using R version 3.5.2.

528 Gene expression profiling

Ten replicate cultures of PA14 BIM2 and 5 of WT were grown in microcosms of $6 \mathrm{~mL} \mathrm{LB}$ media and standardized to an optical density of $0.5 \mathrm{OD} 600$ (approx. $2 \mathrm{X} 10^{\wedge} 9 \mathrm{CFU} / \mathrm{mL}$ ).

531 Five cultures of BIM2 and 5 cultures of WT were inoculated with 8 X $10^{\wedge} 9 \mathrm{PFU}$ (in $500 \mathrm{uL}$ )

532 DMS3vir (MOI 0.5) and 5 cultures of BIM2 remained uninoculated as controls. After 35

533 minutes, 1 hour and 2 hours, $1.5 \mathrm{~mL}$ of cells were pelleted and snap-frozen at $-80 \mathrm{C}$. For

534 RNA extraction, $1 \mathrm{~mL}$ of TRIzol reagent was added immediately after removal from freezer.

535 BCP was used as a phase separator followed by PureLink RNA extraction (Invitrogen) that

536 included a DNAse treatment step. Sample quantities, purities and size distribution were

537 quantifed by Qubit, nanodrop and TapeStation respectively. 150bp libraries were prepared

538 with the TruSeq directional kit and sequenced on an Illumina NovaSeq at Exeter University 539 sequencing centre.

541 The resulting reads were quality filtered using Sickle (default settings, version 1.33) with 542 approx. 6 million read pairs per sample remaining. These were subsequently mapped to the 
resulting read alignments were assigned to genomic features and counted using HTSeq the DESeq2 package (Love et al. 2014).

\section{ProteaseI expression}

550 The proteaseI gene from DMS3vir was cloned into the pHERD30T vector under control of an arabinose inducible promoter. Primers used for cloning are available in table S3. Colonies were selected using a gentamycin marker and blue/white colony screening in a background of dH5-alpha cells (New England Biolabs, UK). The resulting construct was transformed into PA14 and the clone used for assays was verified by Sanger sequencing (University of Sheffield, UK). Optical density was recorded during a 24-hour growth curve at $37 \mathrm{C}$ in LB supplemented with $1 \%$ arabinose. Growth curves of the clone expressing proteaseI and an empty vector control were recorded. Growth rate during log-phase was extracted from the growth curves in $\mathrm{R}$ (version 3.5.2).

\section{Competition assays}

561 For each population, a single clone of each phenotype (CRISPR, SM, Sens.) was picked

562 (where possible) and competed against a LacZ marked reference strain. Populations were 563 serially transferred with a 1:100 dilution in M9 media supplemented with $0.2 \%$ glucose.

564 Populations were plated at $\mathrm{T}=0$ and after 3 days on LB agar supplemeted with $30 \mu \mathrm{g}$ of X-

565 gal to determine the relative frequencies of the clone and the reference. Selection coefficients 566 were determined as described in (van Houte et al. 2016). 
570 Sequence data is available from the European Nucleotide Archive under study number PRJEB31514.

\section{Author contributions}

573 E.R.W., S.v.H., S.M. and A.B. designed experiments. L.C. and S.M. performed the

574 experiments under supervision of S.v.H. and E.R.W. E.O. prepared samples for sequencing.

575 S.M., L.L., A.Biswas. performed sequencing data analyses. E.R.W. and S.M. wrote the 576 manuscript.

\section{Acknowledgements}

E.R.W. and A.B. acknowledge the Natural Environment Research Council, and the

580 Biotechnology and Biological Sciences Research Council for funding. E.R.W further

581 acknowledges the Wellcome Trust and the European Research Council for funding. A.B

582 further acknowledges the Royal Society, the Leverhulme Trust, and the AXA research fund

583 for funding. SVH acknowledges funding from BBSRC (grant number BB/R010781/1). Whole

584 genome sequencing was provided by MicrobesNG (http://www.microbesng.uk) which is

585 supported by the BBSRC (grant number BB/L024209/1). RNA-sequencing was conducted at

586 the University of Exeter sequencing centre which is supported by the Wellcome Trust.

References

589 Alseth EO, Pursey E, Luján AM et al. (2019) Bacterial biodiversity drives the evolution of CRISPR-based phage resistance. Nature, 574, 549-552.

591 Altschul SF, Gish W, Miller W et al. (1990) Basic local alignment search tool. Journal of 592 Molecular Biology, 215, 403-410.

593 Anders S, Pyl PT, Huber W (2015) HTSeq--a Python framework to work with high- 
Balogh B, Jones JB, Iriarte FB, Momol MT (2010) Phage therapy for plant disease control.

Bankevich A, Nurk S, Antipov D et al. (2012) SPAdes: a new genome assembly algorithm and its applications to single-cell sequencing. Journal of computational biology, 19, 455-77.

Bolger AM, Lohse M, Usadel B (2014) Trimmomatic: a flexible trimmer for Illumina sequence data. Bioinformatics, 30, 2114-2120.

Bondy-Denomy J, Pawluk A, Maxwell KL, Davidson AR (2012) Bacteriophage genes that inactivate the CRISPR/Cas bacterial immune system. Nature, 493, 429-432.

Budzik JM, Rosche WA, Rietsch A, O'Toole GA (2004) Isolation and characterization of a generalized transducing phage for Pseudomonas aeruginosa strains PAO1 and PA14. Journal of bacteriology, 186, 3270-3.

Cady KC, Bondy-Denomy J, Heussler GE, Davidson AR, O'Toole, GA (2012) The CRISPR/Cas adaptive immune system of Pseudomonas aeruginosa mediates resistance to naturally occurring and engineered phages. Am Soc Microbiol. 194, 5728-5738.

610 Canchaya C, Fournous G, Chibani-Chennoufi S, Dillmann M-L, Brüssow H (2003) Phage as agents of lateral gene transfer. Current Opinion in Microbiology, 6, 417-424.

612 Caporaso JG, Kuczynski J, Stombaugh J et al. (2010) QIIME allows analysis of high613 throughput community sequencing data. Nature methods, 7, 335-6.

614 Chabas H, van Houte S, Høyland-Kroghsbo NM, Buckling A, Westra ER (2016)

615 Immigration of susceptible hosts triggers the evolution of alternative parasite defence strategies. Proceedings of the Royal Society B: Biological Sciences, 283, 20160721.

617 Chabas H, Lion S, Nicot A et al. (2018) Evolutionary emergence of infectious diseases in 618 heterogeneous host populations. PLoS biology, 16, e2006738. 
619 Chaudhry WN, Pleška M, Shah NN et al. (2018) Leaky resistance and the conditions for the existence of lytic bacteriophage. PLOS Biology, 16, e2005971.

621 Common J, Morley D, Westra ER, van Houte S (2019) CRISPR-Cas immunity leads to a coevolutionary arms race between Streptococcus thermophilus and lytic phage. Philosophical Transactions of the Royal Society B: Biological Sciences, 374, 20180098. rates of microbial sulfur and carbon cycling in wetland ecosystems. Microbiome, 6, 138.

Daly RA, Roux S, Borton MA et al. (2019) Viruses control dominant bacteria colonizing the terrestrial deep biosphere after hydraulic fracturing. Nature Microbiology, 4, 352-361.

Deatherage DE, Barrick JE (2014) Identification of mutations in laboratory-evolved microbes from next-generation sequencing data using breseq. Methods in Molecular Biology,

Dedrick RM, Guerrero-Bustamante CA, Garlena RA et al. (2019) Engineered bacteriophages for treatment of a patient with a disseminated drug-resistant Mycobacterium abscessus. Nature Medicine, 25, 730-733.

Gurney J, Pleška M, Levin BR (2019) Why put up with immunity when there is resistance: an excursion into the population and evolutionary dynamics of restriction-modification and CRISPR-Cas. Philosophical Transactions of the Royal Society B: Biological Sciences, 374, 20180096.

van Houte S, Ekroth AKE, Broniewski JM et al. (2016) The diversity-generating benefits of a prokaryotic adaptive immune system. Nature, 532, 385-388.

641 the evolutionary downside of CRISPR immunity: bacteria and beneficial plasmids. PLoS genetics, 9, e1003844.

643 Kingwell K (2015) Bacteriophage therapies re-enter clinical trials. Nat Rev Drug Discov, 14, 
Koskella B, Brockhurst MA (2014) Bacteria-phage coevolution as a driver of ecological and evolutionary processes in microbial communities. FEMS microbiology reviews, $\mathbf{3 8}$, 916-31.

Koskella B, Lively CM (2009) Evidence for negative frequency-dependent selection during experimenal coevolution of a freshwater snail and a sterilizing trematode. Evolution, 63, $2213-2221$.

Kutter E, De Vos D, Gvasalia G et al. (2010) Phage Therapy in Clinical Practice: Treatment of Human Infections. Current Pharmaceutical Biotechnology, 11, 69-86.

Labrie SJ, Samson JE, Moineau S (2010) Bacteriophage resistance mechanisms. Nature reviews. Microbiology, 8, 317-27.

Gonzalez-Zorn, B, Poyart, C, Rosinski-Chupin, I and Glaser, P (2012) The highly dynamic

CRISPR1 system of Streptococcus agalactiae controls the diversity of its mobilome. Molecular microbiology, 85, 1057-1071.

Love MI, Huber W, Anders S (2014) Moderated estimation of fold change and dispersion for RNA-seq data with DESeq2. Genome Biology, 15, 550.

Mahé F, Rognes T, Quince C et al. (2015) Swarm v2: highly-scalable and high-resolution amplicon clustering. PeerJ, 3, e1420.

Marraffini LA (2015) CRISPR-Cas immunity in prokaryotes. Nature, 526, 55-61.

Martin M (2011) Cutadapt removes adapter sequences from high-throughput sequencing reads. EMBnet.journal, 17, 10-12. elements that confer immunity to phage. Nature Communications, 4, 1430.

668 Paterson S, Vogwill T, Buckling A et al. (2010) Antagonistic coevolution accelerates 
molecular evolution. Nature, 464, 275-278.

Roach DR, Leung CY, Henry M et al. (2017) Synergy between the Host Immune System and

Rose T, Verbeken G, Vos D De et al. (2014) Experimental phage therapy of burn wound infection: difficult first steps. International Journal of Burns and Trauma, 4, 66.

Shipman S, Nivala J, Macklis J, et al. (2017) CRISPR-Cas encoding of a digital movie into the genomes of a population of living bacteria. Nature, 547, 345 .

Shipman S, Nivala J, Macklis J, et al. (2016) Molecular recordings by directed CRISPR spacer acquisition. Science, 353, aaf1175.

Stanley SY, Borges AL, Chen K-H et al. (2019) Anti-CRISPR-Associated Proteins Are

Chao A. (1984) Nonparametric estimation of the number of classes in a population.

Suttle CA (2007) Marine viruses - major players in the global ecosystem. Nature Reviews

Tyson GW, Banfield JF (2007) Rapidly evolving CRISPRs implicated in acquired resistance of microorganisms to viruses. Environmental Microbiology, 10, 200-207. therapy reduces Campylobacter jejuni colonization in broilers. Veterinary Microbiology, 109, 275-283.

Waters E, Neill D, Kaman B et al. Phage therapy is highly effective against chronic lung infections with Pseudomonas aeruginosa. Thorax, 72, 666-667. 
694 Weissman J, Holmes R, Barrangou R et al. (2018) Immune loss as a driver of coexistence

695 during host-phage coevolution. The ISME journal, 12, 585.

696 Westra ER, van Houte S, Oyesiku-Blakemore S et al. (2015) Parasite Exposure Drives

697 Selective Evolution of Constitutive versus Inducible Defense. Current biology, 25,

$698 \quad 1043-1049$.

699 Wright A, Hawkins CH, Anggård EE, Harper DR (2009) A controlled clinical trial of a

700 therapeutic bacteriophage preparation in chronic otitis due to antibiotic-resistant

701 Pseudomonas aeruginosa; a preliminary report of efficacy. Clinical otolaryngology, 34,

$702 \quad 349-57$.

703 Zhang J, Kobert K, Flouri T, Stamatakis A (2013) PEAR: a fast and accurate Illumina Paired-

$704 \quad$ End reAd mergeR. Bioinformatics, 30, 614-620.

705

706

707

708

Supplementary Information

709

710

711

712 


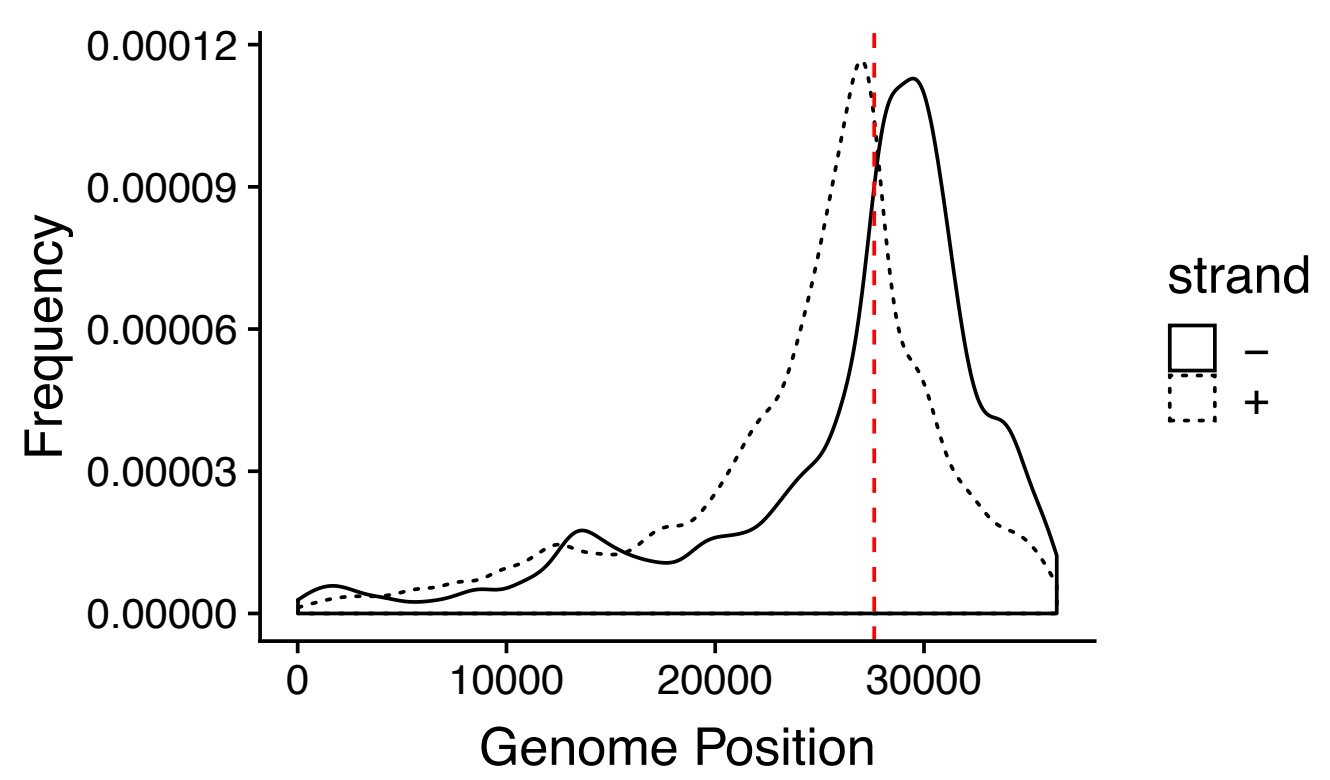

714 Figure S1.

715 Locations of extracted spacers from the CRISPR arrays when mapped to the DMS3vir

716 genome. Red line denotes the location of the priming spacer (CRISPR2 spacer 1).

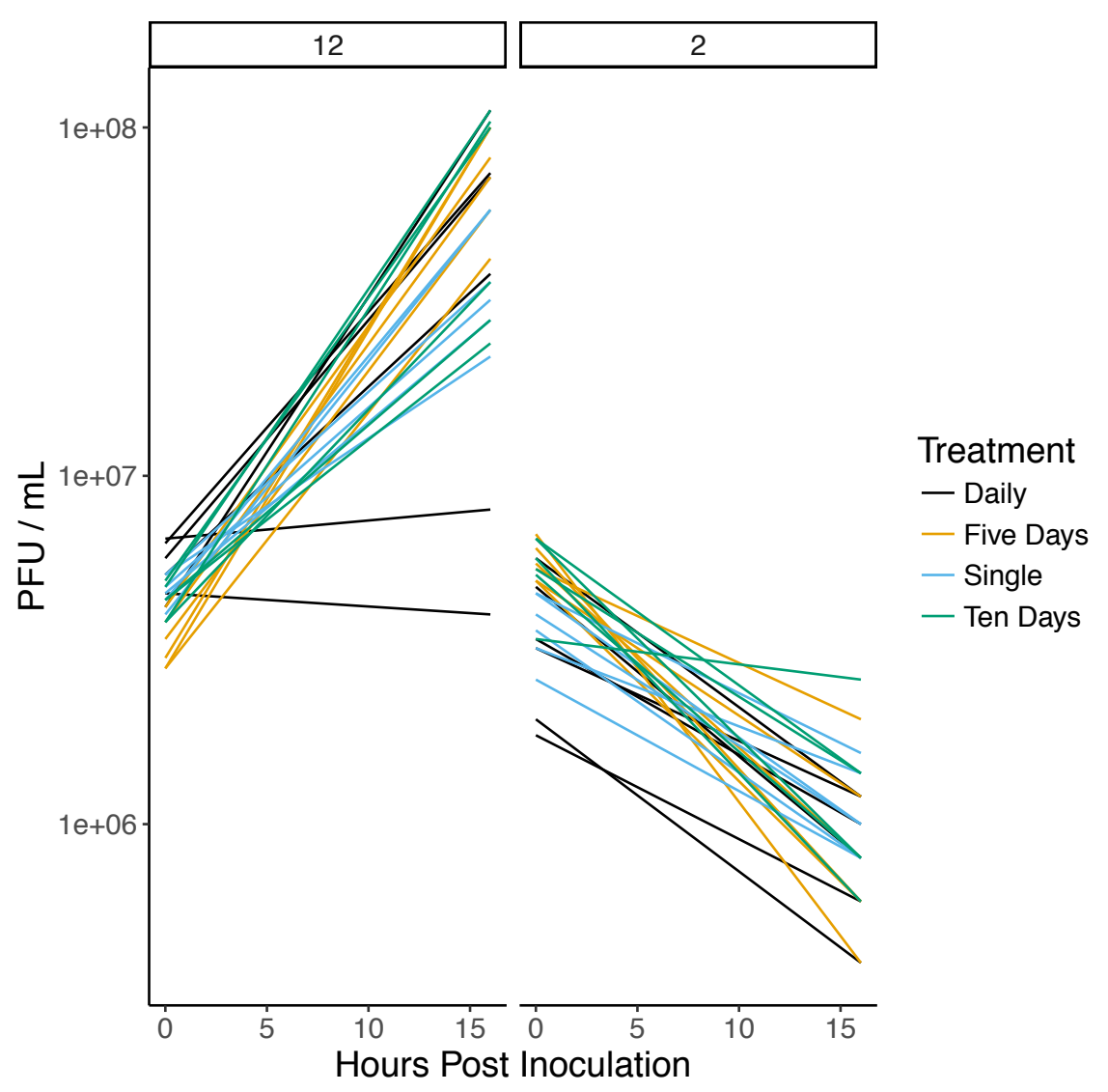


$721 \quad$ Figure S2.

722 Changes in phage titres upon infection with $10^{7}$ PFU DMS3vir of populations isolated from

723 day 12 (left panel, $n=24$ ) or day 2 (right panel, $n=24$ ) from each of the different reinfection

724 regimes. Colours denote the reinfection regimes where the bacterial populations were derived

725 from: bacterial populations isolated from the single infection regime are shown in blue, those

726 from re-infection every five days are shown in yellow, reinfection every 10 days in green,

727 and daily infection in black.

728

729

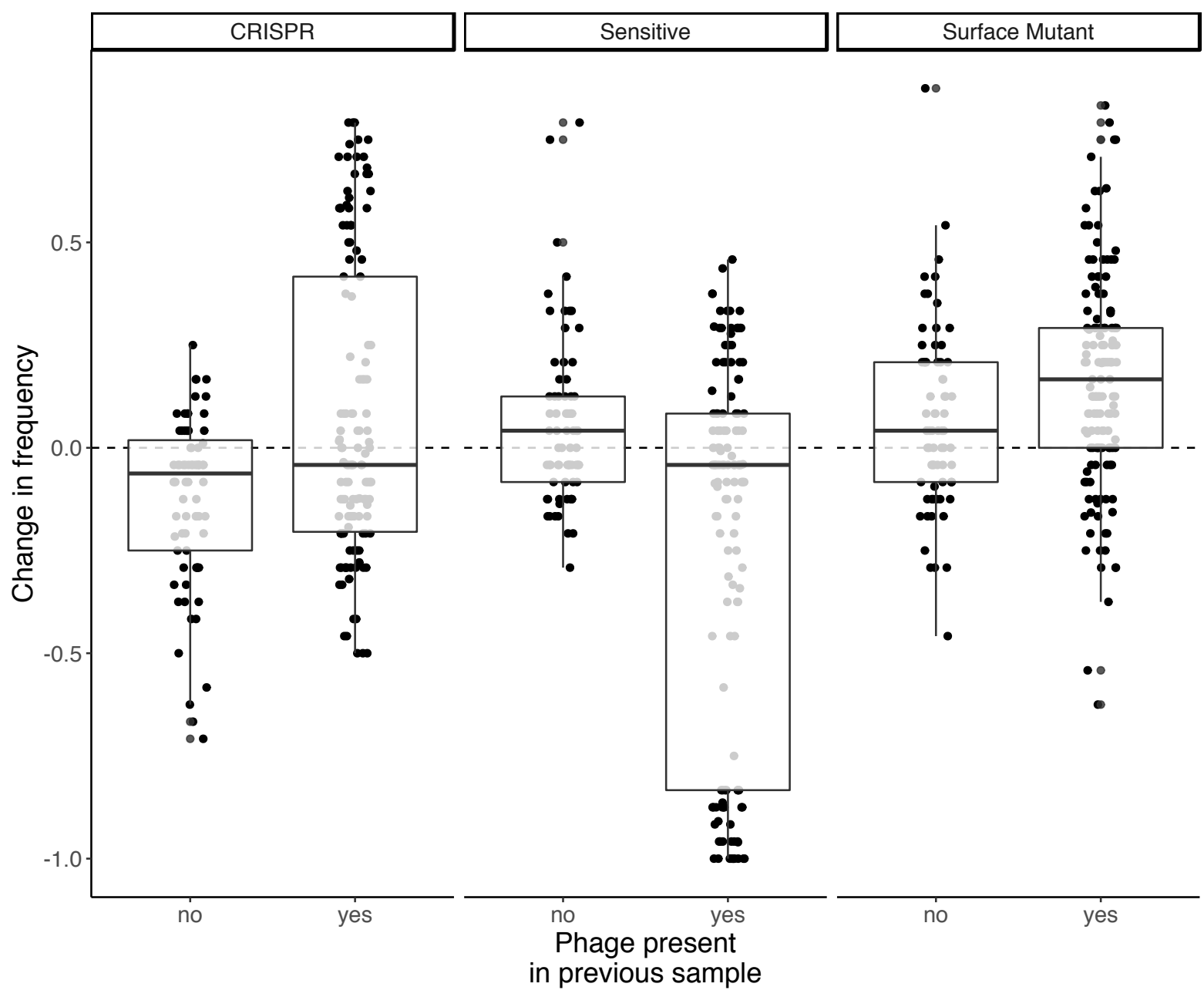


731 Fig. S3. Change in frequency of each phenotype between sampling points during evolution experiment split by presence $(n=402)$ or absence $(n=215)$ of phage in the previous sample.

733 Limit of detection for phage presence is 200 PFU per ml. Boxplots show the median,

734 interquartile range and minimum and maximum values.

735

736

737

738

739

740
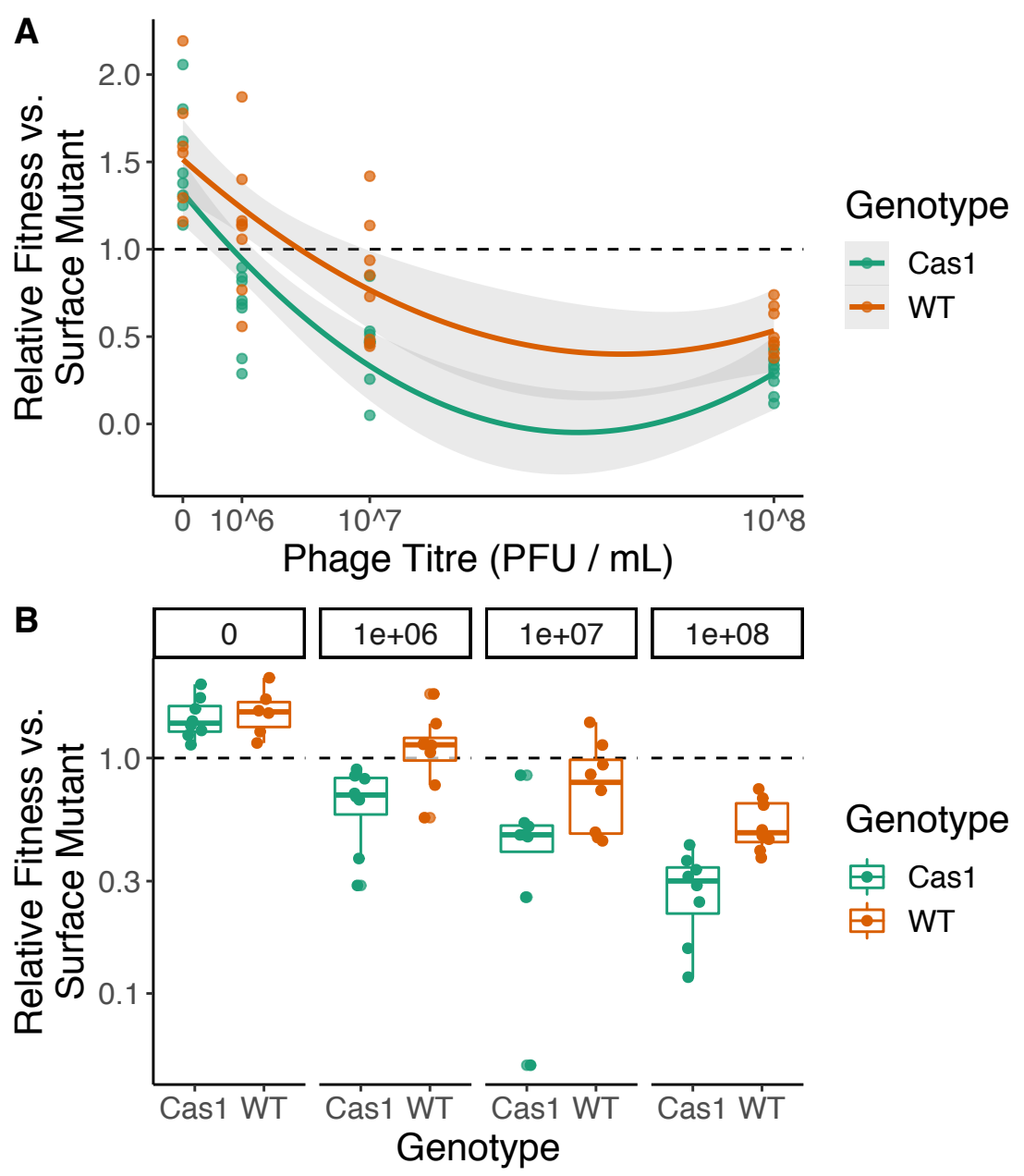

741

742 Figure S4.

743 Relative fitness of bacterial populations with CRISPR-mediated immunity at 3 d.p.i. when

744 competing with a surface mutant. Competitions were carried out between a marked surface 
746 (orange) or a WT-derived strain carrying 2 additional spacers targeting phage DMS3mvir

747 (BIM2; green). Experiments were carried out across a gradient of phage titres, as indicated.

748 The phage used (DMS3mvir) is a mutant of DMS3vir that is targeted by WT PA14. $\mathrm{N}=8$

749 replicates per genotype per phage titre. A) Data presented with phage titre as a continuous

750 variable. B) Data grouped by phage titre to better visualise differences within titres.

751

752

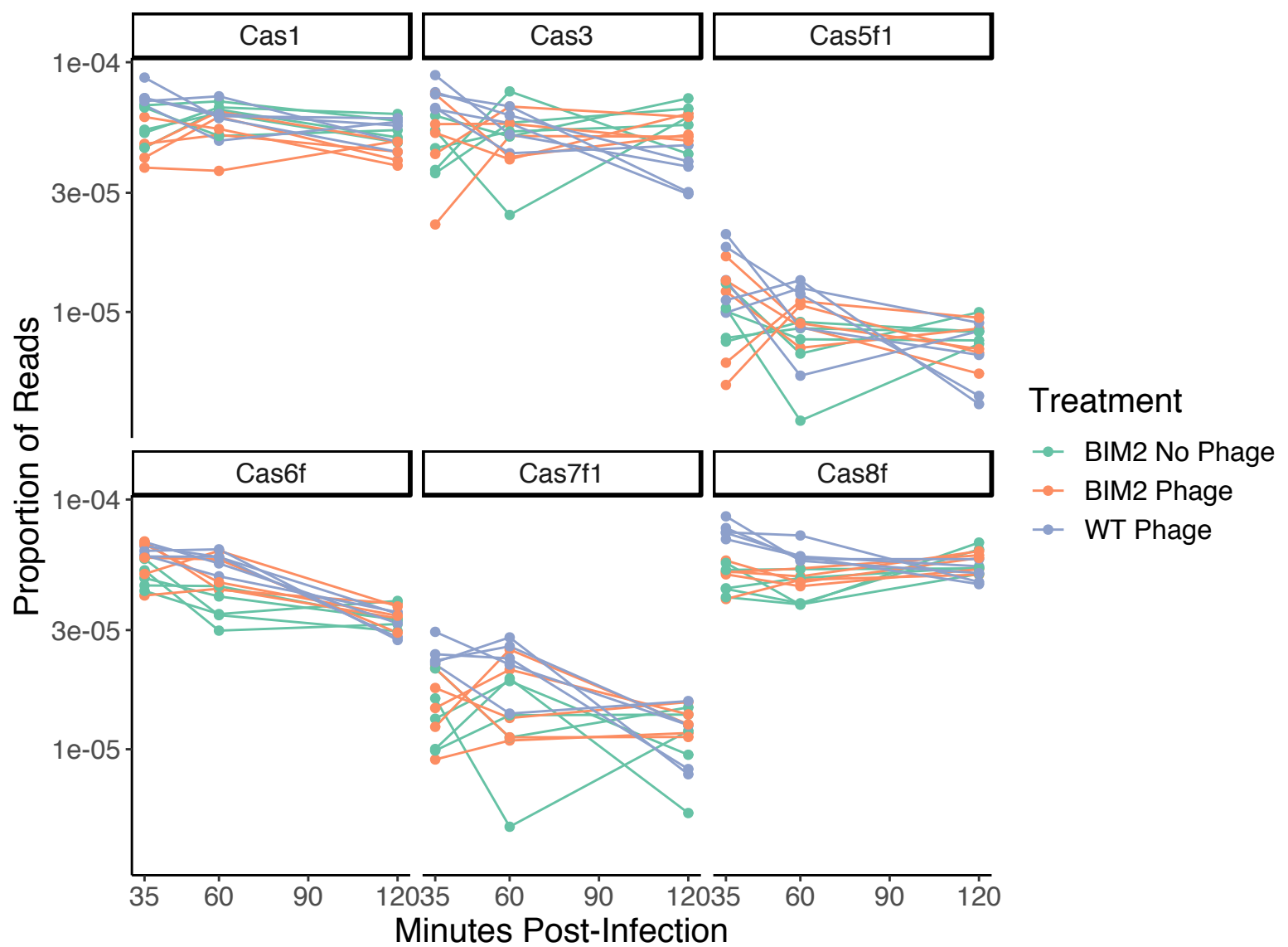

Fig. S5

755 Expression of CRISPR associated genes following infection with 8 X $10^{\wedge} 9$ PFU DMS3vir

756 (MOI 0.5) across PA14 WT (purple, $\mathrm{n}=5$ ), PA14 BIM2 (orange, $\mathrm{n}=5$ ) and PA14 BIM2

757 uninfected controls (green, $\mathrm{n}=5$ ). Samples taken 35, 60 and 120 minutes post-infection. 


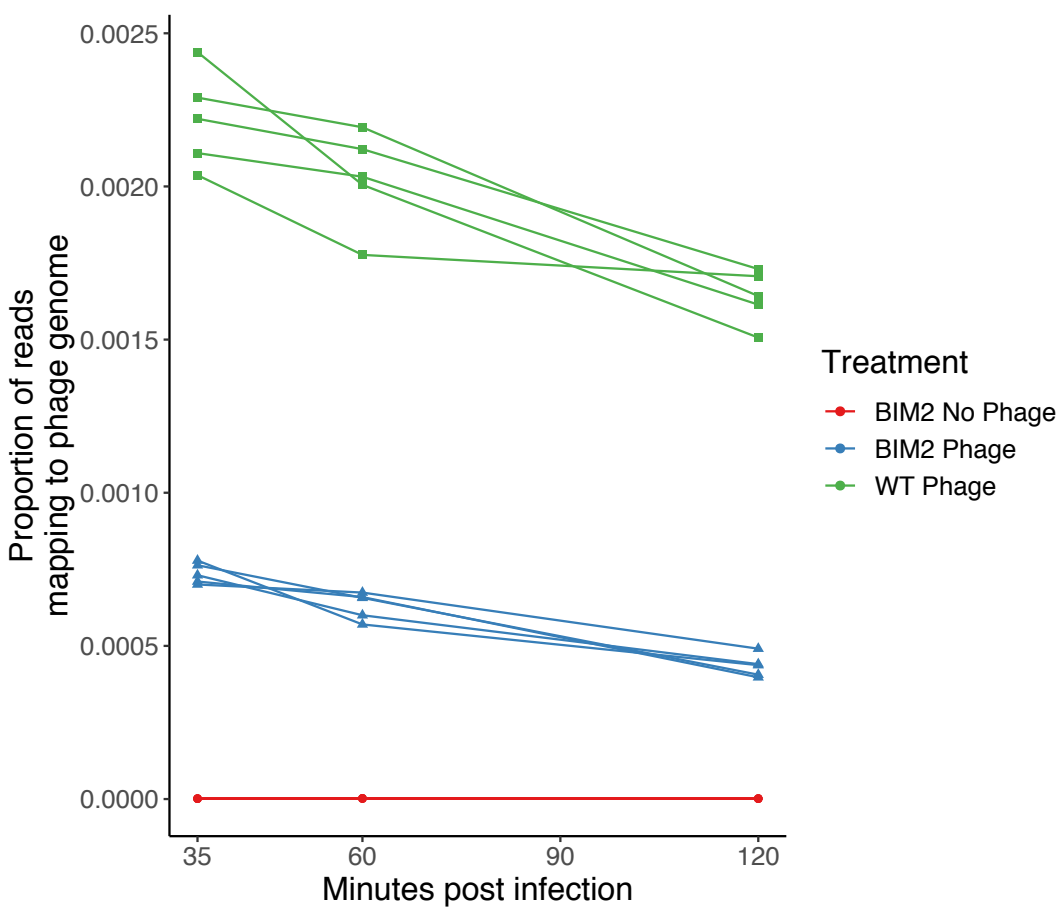

Fig. S6.

763 Total phage gene expression following infection with 8 X 10^9 PFU DMS3vir (MOI 0.5)

764 across PA14 WT (green, $\mathrm{n}=5$ ), PA14 BIM2 (blue, $\mathrm{n}=5$ ) and PA14 BIM2 uninfected

765 controls (red, $\mathrm{n}=5$ ). Samples taken 35, 60 and 120 minutes post-infection. 
A
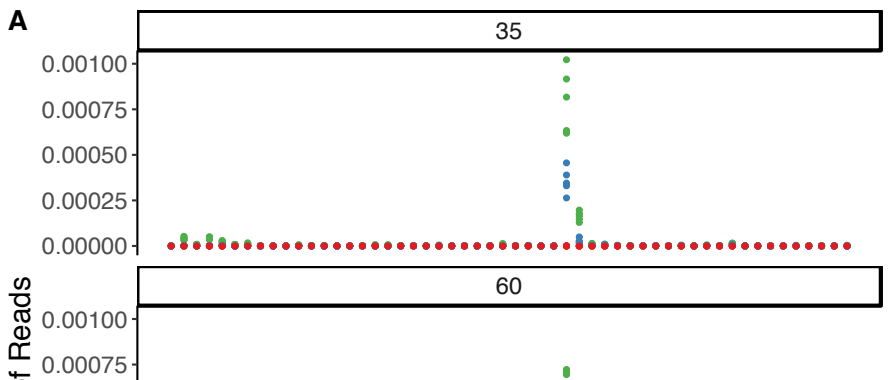

ᄃ 0.00050

은 0.00025

은

믐

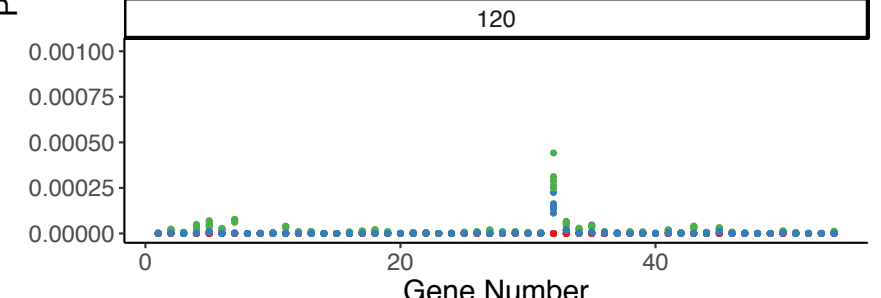

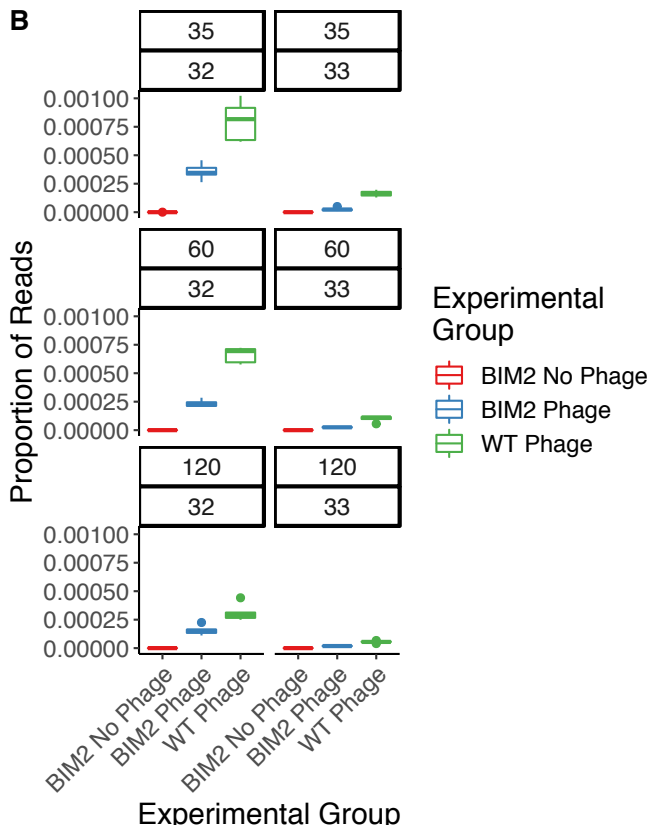

770

Fig. S7

771

A) Phage gene expression of each annotated phage gene following infection with $8 \times 10^{\wedge} 9$

772

PFU DMS3vir (MOI 0.5) across PA14 WT (green, $n=5$ ), PA14 BIM2 (blue, $n=5$ ) and

773

PA14 BIM2 uninfected controls (red, $\mathrm{n}=5$ ) at 35, 60 and 120 minutes post-infection. B)

774

Gene expression of the acr protein (32) and its aca repressor (33) across sampling times and

775

treatments.

776

777

778

779

780

781

782

783

784

785

786

787 
Table S1

Post-hoc comparisons of contrasts that compare relative fitness. Numbers in the contrast

791 denote sampling time (day 4 or day 12) and the given phenotypes (CRISPR, SM or sensitive).

\begin{tabular}{|c|c|c|c|c|c|}
\hline Contrast & Estimate & SE & df & t.ratio & p-value \\
\hline $\begin{array}{l}\text { 4_CRISPR- } \\
\text { 12_CRISPR }\end{array}$ & -0.2773 & 0.0474 & 122.8124 & -5.8475 & $1 e-04$ \\
\hline $\begin{array}{l}\text { 4_CRISPR- } \\
\text { 4_Sens }\end{array}$ & -0.1115 & 0.0586 & 109.4472 & -1.9017 & 0.4067 \\
\hline $\begin{array}{l}\text { 4_CRISPR- } \\
12 \text { Sens }\end{array}$ & -0.3887 & 0.0742 & 111.9035 & -5.2423 & 1e-04 \\
\hline $\begin{array}{l}\text { 4_CRISPR- } \\
\text { 4_SM }\end{array}$ & -0.1243 & 0.0516 & 96.3502 & -2.4093 & 0.1634 \\
\hline $\begin{array}{l}\text { 4_CRISPR- } \\
12 \_ \text {SM }\end{array}$ & -0.4015 & 0.0693 & 111.6448 & -5.7951 & $1 e-04$ \\
\hline $\begin{array}{l}\text { 12_CRISPR- } \\
\text { 4_Sens }\end{array}$ & 0.1658 & 0.0766 & 120.3732 & 2.1639 & 0.2625 \\
\hline $\begin{array}{l}\text { 12_CRISPR- } \\
\text { 12_Sens }\end{array}$ & -0.1115 & 0.0586 & 109.4472 & -1.9017 & 0.4067 \\
\hline $\begin{array}{l}\text { 12_CRISPR- } \\
\text { 4_SM }\end{array}$ & 0.153 & 0.0708 & 113.2484 & 2.1593 & 0.2652 \\
\hline $\begin{array}{l}\text { 12_CRISPR- } \\
\text { 12_SM }\end{array}$ & -0.1243 & 0.0516 & 96.3502 & -2.4093 & 0.1634 \\
\hline $\begin{array}{l}\text { 4_Sens- } \\
12 \_ \text {Sens }\end{array}$ & -0.2773 & 0.0474 & 122.8124 & -5.8475 & $1 e-04$ \\
\hline $\begin{array}{l}\text { 4_Sens- } \\
\text { 4_SM }\end{array}$ & -0.0128 & 0.058 & 108.8156 & -0.2211 & 0.9999 \\
\hline $\begin{array}{l}\text { 4_Sens- } \\
\text { 12_SM }\end{array}$ & -0.2901 & 0.0754 & 119.9723 & -3.8455 & 0.0026 \\
\hline $\begin{array}{l}\text { 12_Sens- } \\
\text { 4_SM }\end{array}$ & 0.2644 & 0.0744 & 112.3086 & 3.5541 & 0.0072 \\
\hline $\begin{array}{l}\text { 12_Sens- } \\
\text { 12_SM }\end{array}$ & -0.0128 & 0.058 & 108.8156 & -0.2211 & 0.9999 \\
\hline 4_SM- & -0.2773 & 0.0474 & 122.8124 & -5.8475 & $1 e-04$ \\
\hline
\end{tabular}

Table S2.

Primers and PCR conditions used for amplicon sequence analysis:

\begin{tabular}{|l|l|l|l|}
\hline CRISPR array & Primers & Sequence & PCR conditions \\
\hline CRISPR 1 & CR1_F & GGCGCTGGAGCCCTTGGGGCTTGG & 95 C X 1 minutes \\
& CR1_R & GCGGCTGCCGGTGGTAGCGGGTG C X 15 & $\begin{array}{l}\text { 95 } \\
\text { seconds } \\
\text { 69 C X 15 } \\
\text { seconds }\end{array}$ \\
\hline
\end{tabular}




\begin{tabular}{|c|c|c|c|}
\hline & & & $\begin{array}{l}72 \text { C X } 30 \\
\text { seconds } \\
72 \text { C X } 7 \text { minutes }\end{array}$ \\
\hline CRISPR 2 & $\begin{array}{l}\text { CR2_F } \\
\text { CR2_R }\end{array}$ & $\begin{array}{l}\text { GCTCGACTACTACAACGTCCGGC } \\
\text { GGGTTTCTGGCGGGAAAAACTCGG }\end{array}$ & $\begin{array}{l}95 \text { C X } 1 \text { minutes } \\
95 \text { C X } 15 \\
\text { seconds } \\
69 \text { C X } 15 \\
\text { seconds } \\
72 \text { C X } 30 \\
\text { seconds } \\
72 \text { C X } 7 \text { minutes }\end{array}$ \\
\hline
\end{tabular}

Table S3

801

Primers for cloning proteaseI gene of DMS3vir

\begin{tabular}{|l|l|}
\hline Primer Name & Sequence \\
\hline ProI_F & 5'GCCGGAATTCGATGGAAAAGAACCGCCTACTCG \\
\hline ProI_R & 5'GCCGGAATTCGATGGAAAAGAACCGCCTACTCG \\
\hline
\end{tabular}

804

805

806 